\title{
RA-Management-Empfehlungen
}

\section{Was ist neu?}

Zum zweiten Mal wurden die Richtlinien der Europäischen Rheumaliga EULAR zur Behandlung der rheumatoiden Arthritis (RA) überarbeitet. Nach 2014 wurden nun 2017 die neuen Empfehlungen publiziert [1]. Das Dokument ist ähnlich lang wie die Vorgängerversion und die Figuren sehen ähnlich aus. Es stellt sich die Frage: Was ist neu?

Bevor diese Frage beantwortet wird, ist es wichtig festzuhalten, was konstant geblieben ist und immer noch gültig ist. Zum einen bleibt Treatto-Target das grundlegende Behandlungskonzept der RA, und das "Target" Remission ist seit 2010 auch durch Kriterien der EULAR und ACR klar definiert. Weiters gibt es keine Änderungen bei dem Umstand, dass Biologika nicht in der Erstlinientherapie und immer in Kombination mit synthetischer Basistherapie eingesetzt werden sollen. Nach Versagen von Methotrexat entscheiden weiterhin prognostisch ungünstige Faktoren darüber, ob ein anderes synthetisches Medikament oder eben ein Biologikum begonnen werden soll.

D) Treat-to-Target bleibt das grundlegende Behandlungskonzept der RA

\section{Erstlinien-Monotherapie}

Was ist nun neu? In der Erstlinientherapie gibt es tatsächlich einige Neuerungen. Hier haben sich die Empfehlungen klar wegbewegt von einer initialen Kombinationstherapie synthetischer Basistherapien, wie sie optional in vorangegangenen Versionen angegeben wurden, $z$. B. die umstrittene Triple-Therapie von Methotrexat, Sulfasalazin und Hydroxychloroquin. Dieser Entscheidung liegen Studien wir "CareRA" zugrunde [2]. Ein klares Bekenntnis der EULAR zur Monotherapie synthetischer Basistherapien also.
Monotherapie jedoch nur mit Einschränkungen, da gleichzeitig die Wertigkeit der initialen Glukokortikoidtherapie - sogar sehr deutlich - gehoben wurde. Die Empfehlung geht auf eine Kombination in allen Fällen (sofern keine Kontraindikationen dagegen sprechen) und nicht mehr auf den optionalen Einsatz. Gleichzeitig wird hervorgehoben, dass die kurzzeitige Therapie entscheidend ist und nicht so sehr die niedrig-dosierte. So kann z. B. ein einmaliger hochdosierter Bolus von Methylprednisolon über ein halbes Jahr Effekte zeigen, die ähnlich einem TNF-Inhibitor sind [3].

》) Die EULAR gibt ein klares Bekenntnis zur Monotherapie synthetischer Basistherapien

\section{Position der JAK-Inhibitoren}

Die letzte wichtige und entscheidende Änderung im Algorithmus bezieht sich auf die Position der JAK-Inhibitoren im Algorithmus. Waren die "JAKinibs" in der letzten Version noch in der Phase III des Algorithmus angesiedelt, also nach dem Einsatz von Biologika, sind diese in der aktuellen klar nach vorne gerückt, nämlich nach Versagen von Phase I, praktisch auf Augenhöhe mit den Biologika. Lediglich die größere Erfahrung von Rheumatologen mit den existierenden Biologika kann diesen den Vorzug („„current practice") gegenüber den neueren Jakinibs geben.

\section{Fazit}

Es hat sich also doch etwas getan im Management der RA. Die Strategie bleibt im Wesentlichen unverändert, jedoch haben sich die Details zum Einsatz verschiedener Therapeutika im Algorithmus zum Teil deutlich verändert bzw. bewegt.

\section{Priv.-Doz. Dr. Daniel Aletaha}

\section{Literatur}

1. Smolen J et al (2017) Ann Rheum Dis 76:960-977

2. Verschueren et al (2015) Ann Rheum Dis 74:27-23

3. IDEA Studie; Nam J et al (2014) Ann Rheum Dis 73:75-85

rheuma plus 2018 $17: 7$

https://doi.org/10.1007/s12688-

018-0162-6

(c) Springer-Verlag GmbH Austria, ein Teil von Springer Nature 2018

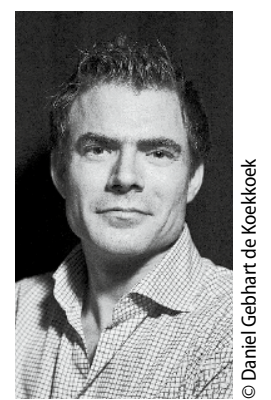

Priv.-Doz. Dr. Daniel Aletaha, Klinische Abteilung für Rheumatologie, Medizinische Universität Wien

Erstveröffentlichung im skriptum Kongressjournal 11/2017: 5-6
Hier steht eine Anzeige.

$$
\text { Springer }
$$

